\section{Arthralgia Associated with Autoimmune Abnormalities under Aromatase Inhibitor Therapy: Outcome after Cessation of Treatment}

\section{To the Editor:}

Arthralgia is a frequent occurrence in patients treated with aromatase inhibitors (AI) for breast cancer. The joint pain is sometimes invalidating and affects treatment compliance ${ }^{1}$. In 2007, we reported a series of 23 patients presenting with arthralgia, sometimes with arthritis, associated with sicca syndrome and with autoimmune abnormalities [antinuclear antibodies (ANA), rheumatoid factor (RF), antithyroglobulin antibodies (ATGA) $]^{2}$. We wished to review these patients after the cessation of AI treatment to determine whether the joint pain persisted and to observe the outcome of the immunological abnormalities.

Twenty-three patients with arthralgia were contacted who had received AI therapy for 5 years (only 17 patients were reviewed in our current study: anastrozole 7, exemestane 5, letrozole 5) and who had, at the initial consultation, either ANA above 1:320 or RF or ANA above 1:160 associated with ATGA. These women had discontinued their AI treatment for at least 1 year. The patients underwent a detailed interview and rheumatological examination, with measurement of ANA, RF, anticyclic citrullinated peptide antibodies (anti-CCP), and ATGA.

The Shapiro-Wilk test was used to verify the normality of continuous data. A paired Wilcoxon signed-rank test was performed to compare ANA under therapy in each group with or without resolution because variables were not Gaussian. ANA was considered an ordinal variable. Fisher's exact test was used to test the relationship between pain evolution and ANA evolution.

Of the 23 patients contacted, 17 were seen again in consultation, 4 were lost to followup, and 2 had had a recurrence of their cancer. The mean age of the 17 patients reviewed was 62.9 years (53-75). Mean time since cessation of AI therapy was 23 months (14-30).

Arthralgia had disappeared in 10 of the 17 patients within a mean of 3 \pm 2 months after AI cessation (Table 1). Two of the 17 patients experienced a decrease in joint pain of over $50 \%$ [mean visual analog scale (VAS) pain 3/10]. Four patients had persistent joint pain mainly affecting the proximal interphalangeal joints, metacarpophalangeal joints, wrists, and shoulders (mean VAS pain 6/10), and 2 had palpable synovitis and persistent sicca syndrome. One patient had persistent sicca syndrome without joint pain. The improvement was not related to the type of molecule administered.
ANA levels decreased in 6 of 15 patients, remained stable in 6 , and increased in 3 patients. RF levels decreased in the 7 patients who initially presented with this abnormality, and became negative in 4. Antithyroperoxidase or ATGA remained at the same levels in all the 4 patients in whom they had been present at the initial consultation. Tests for anti-CCP were negative in all patients and specific ANA were negative in 15 patients. One patient had ACA and 1 had anti-SSA.

Hand radiographs were always normal.

The resolution of arthralgia was associated with a decrease or disappearance of RF in the patients in whom it was present.

Statistical tests should be considered with caution because of the small number of patients. ANA levels during treatment did not affect evolution. ANA positivity was built considering $1 / 160$ as a negative value. Among patients without resolution, 4/7 patients were ANA-positive while under therapy and 3/7 after cessation. Among patients with resolution, 8/10 patients were ANA-positive while under therapy and 4/10 after cessation (p $=0.236$, Fisher's exact test).

There was no correlation between the VAS pain and ANA levels $(\mathrm{p}=0.4)$.

In a previous cohort study of 135 women, arthralgia in AI therapy was classified as diffuse pain, neuropathic pain, tendinitis, or joint pain ${ }^{3}$. In this case, pain may be a worsening of pain in the joints that are classically affected by osteoarthritis or pain that is truly inflammatory in nature, accompanied by slight swelling and morning stiffness affecting the fingers and wrists in particular. The 23 patients that we reported in 2007 presented with this inflammatory pain, often associated with sicca syndrome characterized by the dryness of the mouth and eyes, and with immunological abnormalities. We considered that this pain was not related only to the estrogen deprivation resulting from AI therapy, but was secondary to autoimmune abnormalities probably induced or promoted by the AI therapy and/or the cancer. An aromatase knockout mouse model, which spontaneously develops renal and salivary gland lesions similar to those described in Sjögren syndrome, could confirm this hypothesis ${ }^{4}$. Other authors have since reported cases of rheumatoid arthritis or Sjögren syndrome occurring with AI therapy ${ }^{5,6}$.

In our patients, in two-thirds of cases, joint pain disappeared or declined when AI therapy was discontinued. Conjointly, RF decreased or became negative, unlike ANA whose level fluctuated, and antithyroid antibodies, which persisted. In a few patients, AI therapy could promote the occurrence of autoimmune disease, persisting despite discontinuation of treatment.

Table 1. Clinical and immunological course of 17 patients who presented with arthralgia and autoimmune abnormalities under AI therapy.

\begin{tabular}{|c|c|c|c|c|}
\hline Patient & Age, Yrs & Clinical Course of Arthralgia & Autoantibodies under AI Therapy & Autoantibodies after Cessation \\
\hline 1 & 69 & Resolution & ANA $1 / 320$, RF 72 IU/ml & ANA 1/320, RF $60 \mathrm{IU} / \mathrm{ml}$ \\
\hline 2 & 75 & No clinical improvement & ANA $1 / 320$ & ANA $1 / 640$ \\
\hline 3 & 58 & Resolution & ANA 1/320, RF 244 IU/ml & ANA 1/160, RF 84 IU/ml \\
\hline 4 & 72 & Resolution & ANA 1/320, ATGA 220 IU/ml & ANA 1/160, ATGA 240 IU/ml \\
\hline 5 & 53 & Resolution, sicca syndrome & ANA 1/640, ATGA 335 IU/ml & ANA 1/2500, ATGA $290 \mathrm{IU} / \mathrm{ml}$ \\
\hline 6 & 58 & Resolution & ANA 1/160, ATGA 320 IU/ml & ANA 1/160, ATGA 315 IU/ml \\
\hline 7 & 71 & No clinical improvement, sicca syndrome & ANA $1 / 320$, ACA & ANA $1 / 320$, ACA \\
\hline 8 & 61 & Resolution & $\mathrm{RF} 80 \mathrm{IU} / \mathrm{ml}$ & RF 5 IU/ml \\
\hline 9 & 55 & $70 \%$ improvement & ANA $1 / 320$ & ANA $1 / 160$ \\
\hline 10 & 60 & No clinical improvement & ANA $1 / 320$, RF $44 \mathrm{IU} / \mathrm{ml}$ & ANA $1 / 320$, RF 0 IU/ml \\
\hline 11 & 62 & Resolution & ANA 1/160, ATGA 257 IU/ml & ANA 1/160, ATGA 255 IU/ml \\
\hline 12 & 71 & Resolution & ANA $1 / 2500$ & ANA $1 / 640$ \\
\hline 13 & 59 & Resolution & ANA $1 / 320$ & ANA $1 / 160$ \\
\hline 14 & 56 & Resolution & ANA $1 / 320$, RF 77 IU/ml & ANA $1 / 640$, RF 8 IU/ml \\
\hline 15 & 62 & $80 \%$ improvement & ANA 1/160, RF $132 \mathrm{IU} / \mathrm{ml}$ & ANA $1 / 160$, RF 0 IU/ml \\
\hline 16 & 71 & No clinical improvement, sicca syndrome & ANA $1 / 640$, anti-SSA AB 58 & ANA 1/160, anti-SSA AB 30 \\
\hline 17 & 56 & No clinical improvement & $\mathrm{RF} 38 \mathrm{IU} / \mathrm{ml}$ & RF 0 IU/ml \\
\hline
\end{tabular}

AI: aromatase inhibitor; ANA: antinuclear antibodies; RF: rheumatoid factor; ATGA: antithyroglobulin antibodies; ACA: anticentromere antibodies.

$$
\text { Personal non-commercial use only. The Journal of Rheumatology Copyright (C) 2016. All rights reserved. }
$$


MICHEL LAROCHE, MD, Centre de Rhumatologie, Hôpital Pierre-Paul Riquet; MARIE SENIOW, MD, Centre de Rhumatologie, Hôpital Pierre-Paul Riquet; HENRI ROCHÉ, MD, Institut Universitaire du Cancer Toulouse - Oncopole; ADELINE RUYSSEN-WITRAND, MD, Centre de Rhumatologie, Hôpital Pierre-Paul Riquet, Toulouse, France. Address correspondence to Professor M. Laroche, Centre de Rhumatologie, Hôpital Pierre-Paul Riquet, 1 place du Dr Baylac, 31059 Toulouse Cedex, France.E-mail: laroche.m@chu-toulouse.fr

\section{REFERENCES}

1. Niravath P. Aromatase inhibitor-induced arthralgia: a review. Ann Oncol 2013;24:1443-9.

2. Laroche M, Borg S, Lassoued S, De Lafontan B, Roché H. Joint pain with aromatase inhibitors: abnormal frequency of Sjögren's syndrome. J Rheumatol 2007;34:2259-63.

3. Laroche F, Coste J, Medkour T, Cottu PH, Pierga JY, Lotz JP, et al. Classification of and risk factors for estrogen deprivation pain syndromes related to aromatase inhibitor treatments in women with breast cancer: a prospective multicenter cohort study. J Pain 2014; 15:293-303.

4. Shim GJ, Warner M, Kim HJ, Andersson S, Liu L, Ekman J, et al. Aromatase-deficient mice spontaneously develop a lymphoproliferative autoimmune disease resembling Sjögren's syndrome. Proc Natl Acad Sci U S A 2004;101:12628-33.

5. Morel B, Marotte H, Miossec P. Will steroidal aromatase inhibitors induce rheumatoid arthritis? Ann Rheum Dis 2007;66:557-8.

6. Guidelli GM, Martellucci I, Galeazzi M, Francini G, Fioravanti A. Sjögren's syndrome and aromatase inhibitors treatment: is there a link? Clin Exp Rheumatol 2013;31:653-4.

J Rheumatol 2016;43:10; doi:10.3899/jrheum.160254 